\title{
PRODUCCIÓN DE NÉCTAR Y FLORES COMO RASGOS COMPENSATORIOS AL ROBO DE NÉCTAR EN BOUVARDIA TERNIFOLIA (RUBIACEAE): EXPERIMENTOS DE CAMPO
}

\author{
luis Salinas-Esquivel', María del Coro Arizmendi², César A. Domínguez ${ }^{3}$, \\ Citlalli Castillo-Guevara ${ }^{4}$ y Carlos Lara ${ }^{4,5}$ \\ 'Maestría en Ciencias Biológicas, Facultad de Ciencias, Universidad Nacional Autónoma de México, \\ México, D.F., México \\ ${ }^{2}$ Laboratorio de Ecología, UBIPRO, FES-Iztacala, Universidad Nacional Autónoma de México, \\ Tlanepantla, Estado de México, México \\ ${ }^{3}$ Instituto de Ecología, Universidad Nacional Autónoma de México, México, D.F., México \\ ${ }^{4}$ Centro de Investigación en Ciencias Biológicas, Universidad Autónoma de Tlaxcala, Tlaxcala, Tlaxcala, México \\ ${ }^{5}$ Autor para la correspondencia: laracar@posgradouatx.com.mx
}

\begin{abstract}
Resumen: El néctar de flores polinizadas por colibríes es usualmente robado por animales no polinizadores, por ello se esperaría que plantas sometidas al robo de néctar presenten distintas estrategias que permitan mitigar los costos asociados. En este estudio, evaluamos la producción de néctar y flores como rasgos para mitigar el robo de néctar en la planta diestílica Bouvardia ternifolia (Rubiaceae) en el Parque Nacional la Malinche, Tlaxcala, México. Plantas de ambos morfos fueron sometidas a tres tratamientos: (1) tratamiento de remoción de néctar, (2) tratamiento sin remoción y (3) tratamiento control (plantas sin exclusión ni extracción de néctar). Durante el periodo de floración, cuantificamos semanalmente el volumen de néctar (15 flores/planta) y el número de flores producidas por planta. Polinizamos manualmente las flores focales y los frutos producidos se recolectaron y sus semillas contadas. Las flores con y sin remoción de néctar produjeron similar recompensa; contrario a los pequeños volúmenes producidos en flores control. Las flores del morfo pin mostraron mayores volúmenes y concentraciones de néctar que las thrum. No encontramos efecto de los tratamientos sobre la producción de flores en ambos morfos. Las flores con y sin remoción de néctar produjeron más semillas que las flores control. Nuestros resultados sugieren que Bouvardia puede sobrecompensar el néctar robado, pero no las flores; sin afectar el número de semillas producidas.
\end{abstract}

Palabras clave: Bouvardia ternifolia, producción de flores y néctar, robo de néctar, Tlaxcala.

\begin{abstract}
Nectar of hummingbird-pollinated plants is usually robbed by non-pollinator animals, for that reason it is possible that the robbed plants show different strategies to mitigate the associated costs. In this study, we evaluated the production of nectar and flowers as traits to mitigate the nectar robbing in the distylous plant Bouvardia ternifolia (Rubiaceae) in the National Park La Malinche, Tlaxcala. Plants of both morphs were subject to three different treatments: (1) treatment with nectar removal, (2) treatment without nectar removal, and (3) treatment control. During the flowering period, we weekly quantified the nectar volume (15 flowers/plant) and the number of flowers per plant. We manually pollinated the focal flowers and the produced fruits were collected and their seeds counted. Similar volumes of nectar were produce in the flowers with and without nectar removal; in opposition to the small collected volumes in the control flowers. Long-styled flowers (pin morph) produced more nectar volume and sugar than short-styled flowers (thrum morph). There was not effect of treatments on the flowers production in both morphs. Flowers with and without nectar removal produced more seeds than control flowers. Our results showed that Bouvardia can over-compensate the nectar robbed, but not flowers; without affecting the number produced seeds.
\end{abstract}

Keywords: Bouvardia ternifolia, flower and nectar production, nectar robbing, Tlaxcala.

$\mathbf{L}$ os polinizadores han sido tradicionalmente vistos como una de las principales presiones selectivas en la evolución de las características florales de las plantas que visitan; sin embargo, los visitantes florales ilegítimos, como por ejemplo los robadores de néctar, también son albergados por estas plantas y éstos también pueden influir en la evolución de sus caracteres florales debido a que pueden tener un fuerte impacto en su adecuación (Irwin y Brody, 2000; Maloof y 
Inouye, 2000). Diversos estudios han documentado que las plantas sometidas al efecto de organismos antagonistas tales como herbívoros y patógenos pueden responder a ellos a través de dos estrategias de defensa que permiten minimizar su daño (Strauss y Agrawal, 1999; Roy y Kirchner, 2000; Strauss et al., 2002). Por ejemplo, la síntesis de inhibidores de proteinasas en hojas de tomate (Lycopersicon esculentum) en respuesta a la herbivoría por larvas de lepidóptero. La defensa inducida reduce la intensidad de ataque por estos herbívoros, en comparación con plantas en los cuales la síntesis de estos inhibidores no ocurre (Howe et al., 1996). Este tipo de estrategia es conocida como resistencia, capacidad de disminuir la frecuencia del daño por el consumidor. Por el contrario, la tolerancia, capacidad de las plantas para compensar el daño provocado por el consumidor, puede ser ejemplificada en un estudio realizado en la trompetilla escarlata (Ipomopsis aggregata). En Arizona, Estados Unidos, plantas de esta especie fueron expuestas a altos niveles de herbivoría por mamíferos tales como el venado cola-negra (Odocoileus hemionus) y el alce (Cervus elaphus). Los resultados indicaron que estas plantas produjeron mayor número de inflorescencias y flores que plantas no sometidas a la herbivoría, igualando incluso el número de semillas producidas (Paige y Whitham, 1987).

En estudios recientes se ha planteado la factibilidad de aplicar el concepto de tolerancia a otras interacciones antagonistas, además de la herbivoría, incluyendo el robo de néctar (Irwin et al., 2004, 2008). El robo de néctar efectuado por numerosos organismos podría estar provocando respuestas de defensa en las plantas afectadas, las cuales disminuirían o compensarían de alguna manera el potencial costo energético que representa la producción de néctar y la subsecuente producción de semillas. La tolerancia sería una posible respuesta para disminuir el daño que reciben muchas especies de plantas en su éxito reproductivo, tal como sucede en las interacciones planta-herbívoro y planta-patógeno (Irwin y Brody, 2000; Lara y Ornelas, 2002a, 2003). Además, las especies de plantas afectadas por organismos que roban néctar pueden variar en la capacidad de producir néctar y en sus despliegues florales, y esta variación sugiere que las plantas podrían modificar estos caracteres florales para mitigar los efectos del robo de néctar (Lara y Ornelas 2001a, b; Maloof y Inouye, 2000; Irwin et al., 2008).

Varios estudios han documentado los efectos directos e indirectos que tienen antagonistas como los herbívoros y ladrones de néctar en el arbusto diestílico Bouvardia ternifolia (Rubiaceae). Por ejemplo, se ha probado que la herbivoría tiene efectos negativos en los patrones de producción y calidad de néctar de las plantas afectadas, además de provocar indirectamente una reducción en el número de visitas y los tiempos de llegada de sus colibríes polinizadores, teniendo como consecuencia final un efecto negativo en el número de semillas producidas (Torres-Díaz, 2007; Torres et al., 2008). De la misma forma, se ha documentado que las flo- res sometidas a niveles altos de robo de néctar disminuyen la cantidad y calidad del néctar rellenado, lo cual provoca menor tasa de visitas por los colibríes y afecta negativamente la producción de semillas de estas plantas (Salinas, 2007; Torres et al., 2008). Esta evidencia sugiere que los antagonistas pueden estar modulando indirectamente la naturaleza, fuerza y consecuencias de la interacción entre $B$. ternifolia y sus colibríes polinizadores (Torres et al., 2008).

Los rasgos reproductivos de muchas especies de plantas polinizadas por animales pueden a menudo reflejar un compromiso entre el conflicto de la presión selectiva ejercida tanto por los polinizadores como por organismos antagonistas (Brody y Mitchell, 1997; Strauss y Irwin, 2004; Torres et al., 2008). Es por eso que en el presente trabajo utilizamos dos rasgos no mutuamente excluyentes, que son el néctar y la producción de flores, tal como ha sido propuesto por Irwin et al. (2008) en el único trabajo previo al respecto. Estos autores sugieren que ambos rasgos pueden ser análogos a mecanismos de tolerancia que las plantas utilizan contra los herbívoros: (1) los altos volúmenes de néctar son análogos al alto crecimiento (o producción) para reemplazar la pérdida de recursos producto del consumo foliar, y (2) la alta producción de flores es análoga con la reasignación de recursos vía el despliegue de la reservas acumuladas para reemplazar los daños producidos por el consumo. De esta manera, la recompensa o producción de flores inducidas en respuesta al impacto del robo de néctar (compensación) podría promover la presencia de suficiente néctar residual o flores para la atracción de los polinizadores. Además, amortiguaría el efecto en la reproducción de las plantas, a pesar de las pérdidas provocadas por los robadores de néctar.

La tolerancia a la herbívora es típicamente evaluada usando clones o plantas cercanamente emparentadas (revisión en Strauss y Agrawal, 1999). En nuestro estudio utilizamos plantas creciendo en condiciones silvestres y de relaciones genéticas desconocidas para comprobar si estos rasgos florales pueden relacionarse con una mitigación de los costos de polinización y reproducción provocados por el robo de néctar. En todos los casos, los tratamientos explicados en el método fueron asignados aleatoriamente y realizamos una manipulación artificial de estos rasgos para responder: (1) si el alto grado de robo de néctar se relaciona con una alta producción de flores y de volúmenes de néctar, que pudiera amortiguar los efectos negativos de estos visitantes y (2) si existe una compensación por producción de flores y néctar que pudiera mitigar los costos reproductivos en Bouvardia ternifolia.

\section{Métodos}

Sitio de estudio. El trabajo de campo de este estudio se realizó de junio a octubre del 2009, en el Parque Nacional La Malinche, Tlaxcala (19 6’ N, $19^{\circ} 20^{\prime} \mathrm{O}$; a 2,900 m s.n.m.), México, en un área de parches en floración de Bouvardia 
ternifolia cuya superficie es de aproximadamente 50 ha. La vegetación en esta área natural protegida (45,711 ha) se encuentra compuesta principalmente por bosques de pino, encino y oyamel, y está localizada a $80 \mathrm{~km}$ de la ciudad de Tlaxcala, Tlaxcala. La precipitación media anual es de 800 $\mathrm{mm}$ y la temperatura media anual es de $15^{\circ} \mathrm{C}$. El clima es templado-húmedo la mayor parte del año, con una temporada seca y fría de octubre a marzo. La vegetación está bien conservada y dominada por Abies religiosa (H.B.K) Cham. \& Schlecht, Pinus montezumae Lamb., P. pseudostrobus Lindl, Quercus laurina Humb. \& Bonpl. y Q. crassipes Humb. \& Bonpl. (Díaz-Ojeda, 1992).

Especie estudiada. Bouvardia ternifolia (Cav.) Schltdl. (Rubiaceae) es un arbusto diestílico autoincompatible (a partir de ahora mencionada sólo como Bouvardia), mide de 1-1.5 metros de altura; y está ampliamente distribuido en áreas abiertas de los bosques de Arizona, EUA hasta el sur de México (Faivre, 2000). Las flores presentan las corolas fusionadas, en forma de tubo y agrupadas en cimas terminales. Las flores con estilos largos tienen corolas más cortas que las flores con estilos cortos, provocando la distinción de sus dos morfos, denominados como pin (longiestilados) y thrum (breviestilados) respectivamente. Las flores duran de 4-5 días y cambian de color de rojo escarlata a rojo pálido o rosa en el tercer día. En La Malinche, su periodo de floración es de mayo a agosto y cinco especies de colibríes son sus principales polinizadores: Archilochus colubris, Colibri thalassinus, Eugenes fulgens, Hylocharis leucotis y Lampornis clemenciae (Lara, 2006; Salinas-Esquivel, 2007; Torres-Díaz, 2007; Torres et al., 2008).

Un estudio previo realizado por Hernandez-Guerrero (2006) en la misma localidad analizada en el presente estudio, reporta que los morfos de Bouvardia tienen una producción diferencial de flores y néctar. El morfo thrum produce más flores que el morfo pin $(F=18.36, \mathrm{gl}=1, P=0.0001)$, mientras que el morfo pin presenta una mayor producción de néctar que el morfo thrum (con un máximo de $2 \mu 1$ y un mínimo de $1 \mu \mathrm{l}$ respecto a thrum el cual tuvo un máximo de $1.7 \mu \mathrm{l}$ y un mínimo de $0.5 \mu \mathrm{l}$ ), aunque en lo que respecta a sus concentraciones de néctar y la cantidad de frutos producidos no hubo diferencias entre morfos (HernandezGuerrero, 2006).

Estudios realizados en Chavarrillo, Veracruz y La Malinche, Tlaxcala, han demostrado que las flores de Bouvardia hospedan comúnmente ácaros del género Tropicoseius (Ascidae) los cuales son ladrones de néctar y pueden reducir los volúmenes de recompensa hasta un 50\% (Lara y Ornelas, 2002b; Salinas-Esquivel, 2007).

Evaluación de la compensación de néctar. Cada una de las actividades adelante mencionadas fueron llevadas a cabo en un área de aproximadamente 50 ha en parches en floración de Bouvardia. La respuesta de esta especie diestílica al robo de néctar se determinó a partir de: (1) la respuesta de la plan- ta completa a la remoción o no de néctar de todas sus flores en tres tratamientos (sin remoción, con remoción y control) y (2) en el volumen y concentración de néctar tomado de un grupo de flores por cada tratamiento.

Para determinar si Bouvardia responde al robo de néctar con una sobreproducción del volumen de néctar y/o del azúcar contenido, del 14 de junio al 10 de agosto del 2009 (desde el inicio hasta el final de la floración), se eligieron aleatoriamente y marcaron nueve plantas por morfo $(n=18)$, distribuidas en nuestro sitio de estudio. La plantas seleccionadas se subdividieron a su vez en tres grupos de tres plantas por morfo $(\mathrm{n}=9)$. Cada grupo de tres plantas fue sometido a un tratamiento distinto, los cuales simularon condiciones en que las plantas estuvieran o no sometidas al robo del néctar, como se detalla a continuación: (1) Grupo sin remoción de néctar. A este grupo de plantas se les cubrió totalmente con maya de tul durante todo su periodo de floración y fue untada con Tanglefoot (macilla viscosa; Tanglefoot Co., Grand Rapids, Michigan, EUA) en cada uno de los pedicelos de todas sus flores y tallos para evitar que cualquier visitante rastrero pudiera llegar a ellas y consumir su néctar. (2) Grupo con remoción de néctar. A este grupo de plantas no se le excluyó de ninguna forma y se le extrajo el néctar de todas sus flores tres veces por día $(07: 00,12: 00$ y 16:00 h) durante todo su periodo de floración. Las extracciones fueron realizadas con capilares de $5 \mu 1$ y de forma tal que las flores no recibieran daño por esta acción. (3) Grupo control. Este grupo de plantas no recibió exclusión ni tampoco extracción de néctar.

Posteriormente, una vez establecidos y debidamente marcados cada uno de los grupos de plantas experimentales (tres grupos por morfo), en cada uno de ellos se eligieron a su vez grupos de cinco flores por planta por morfo $(n=90)$ con el objetivo de contrastar el posible efecto de la extracción de néctar entre los tres diferentes grupos en su producción. Con esto, si el robo de néctar promueve una compensación en la producción de néctar, entonces las flores de las plantas cuya extracción es constante (simulando robo), deberían presentar los mayores volúmenes colectados. Para ello, las flores de cada planta experimental perteneciente a cada grupo se eligieron aleatoriamente en botón a punto de abrir, se marcaron y cubrieron con bolsas de tul para evitar la llegada de visitantes que pudieran extraer el néctar. Al siguiente día, ya abiertas las flores, se procedió a medir el volumen de néctar con capilares de $5 \mu \mathrm{l}$; además, se midió la concentración de azúcar con un refractómetro portátil (American optical 10431, Buffalo, Newyork, EUA; rango de concentración $0^{\circ}-50^{\circ}$ escala BRIX), de la siguiente forma para cada grupo: (a) Conjunto de flores del grupo sin remoción de néctar. A las flores elegidas en cada uno de los dos morfos correspondientes a este grupo se les midió el volumen y concentración de néctar acumulado de tres días, edad a la cual los estigmas son receptivos y posteriormente se polinizaron manualmente. (b) Conjunto de flores del grupo 
con remoción de néctar. A cada una de las flores elegidas de este grupo se les midió la concentración y volumen una vez por día (09:00 - 13:00 h), durante tres días y posteriormente fueron polinizadas manualmente. A pesar de que las plantas correspondientes a este grupo no fueron cubiertas con tul, las flores elegidas si permanecieron cubiertas para evitar que el néctar fuera alterado por visitantes florales. (c) Conjunto de flores del grupo control. A las flores que se eligieron en plantas correspondientes a este grupo se les midió diariamente el volumen y concentración de néctar durante tres días consecutivos y posteriormente se polinizaron manualmente, siguiendo el mismo procedimiento que el grupo anterior e igualmente sólo fueron cubiertas con tul las flores utilizadas para las mediciones.

Los tratamientos a, b y c fueron repetidos en dos ocasiones, al principio (del 14 al 18 de junio) y final de la floración (del 6 al 10 de agosto), y todas las mediciones realizadas se llevaron a cabo utilizando micropipetas de $5 \mu \mathrm{l}$, para el volumen, y un refractómetro portátil para la concentración de néctar. Asimismo, el resto de las flores de todos los grupos que no se utilizaron para las mediciones fueron polinizadas manualmente y de forma continua para evitar posibles efectos de limitación de polinización. Para todas estas polinizaciones se utilizó un pincel y se colectó el polen de flores del morfo opuesto y de un sólo individuo por morfo (asegurándose que no estuvieran genéticamente emparentados), a manera de controlar posibles efectos genéticos. Todas las flores polinizadas manualmente permanecieron embolsadas para aislarlas de potenciales polinizadores.

Al final de cada uno de los tratamientos y de la temporada de floración, todos los frutos obtenidos de todas las flores de las plantas utilizadas en todos los tratamientos se colectaron y el número de semillas fue cuantificado. La viabilidad de las semillas colectadas fue corroborada con sal de tetrazolio (Kearns y Inouye, 1993).

Evaluación de la compensación de flores. Para probar experimentalmente si existe una relación entre el nivel de robo tolerado por las plantas de Bouvardia y su producción de flores total, en todas las plantas experimentales se cuantificó el número de flores abiertas una vez por semana (temporada junio-agosto 2009).

Análisis estadísticos. Para comparar el efecto de los tratamientos en los volúmenes y concentraciones de néctar producidos por ambos morfos, tanto al inicio como al final de la floración, se utilizó un ANDEVA de medias repetidas con tres factores: morfo (pin y thrum), temporada (inicio y final de la floración) y tratamiento (sin remoción, con remoción y control). Asimismo, los resultados obtenidos sobre los conteos de flores y de semillas producidas en cada uno de los tratamientos se compararon respectivamente por medio de un ANDEVA de dos factores; donde el número de semillas y flores fueron las variables dependientes mientras el tratamiento y morfo fueron las variables independientes. Pruebas post hoc fueron realizadas para examinar diferen- cias entre morfos y grupos de plantas sometidas a diferentes tratamientos (Zar, 1999).

\section{Resultados}

Compensación de néctar. Los resultados obtenidos demostraron que existen diferencias significativas en la producción de néctar entre morfos $\left(F_{1,84}=33.446, P=0.0001\right)$, entre tratamientos $\left(F_{2,84}=34.324, P=0.0001\right)$ y en la interacción morfo por tratamiento $\left(F_{2,84}=3.238, P=0.0442\right)$. De esta manera, independientemente del tratamiento aplicado, las flores del morfo pin presentaron una mayor producción de néctar. Asimismo, independientemente del morfo, las plantas con tratamiento control fueron las que presentaron una menor producción de néctar. La significancia de la interacción demostró que las flores sometidas a extracción constante producen tanto néctar como aquellas sometidas a exclusión y cuyo néctar se acumuló por tres días. Asimismo, indica que ambos tratamientos difieren del poco volumen cosechado en flores con extracción natural (Figura 1).

Los volúmenes de néctar cuantificados al inicio y final de la temporada de la floración, independientemente del morfo y tratamiento aplicado, indicaron ser mayores al final de la floración $\left(F_{1,84}=3324.553, P=0.0001\right)$. La interacción del factor temporada con morfo fue significativa, producto de los mayores volúmenes cuantificados en el morfo pin al final de la floración $\left(F_{1,84}=27.857, P=0.0001\right)$. Asimismo, se cuantificó un mayor volumen en los tratamientos de remoción cuando fueron aplicados al final de la floración $\left(F_{2,84}\right.$ $=7.884, P=0.0007)$. Finalmente, no se observaron diferencias significativas en la interacción temporada $\times$ tratamiento $\times$ morfo $\left(F_{2,84}=0.574, P=0.565\right)$.

El análisis de las concentraciones de néctar demostró que, independientemente del tratamiento recibido, las plantas de

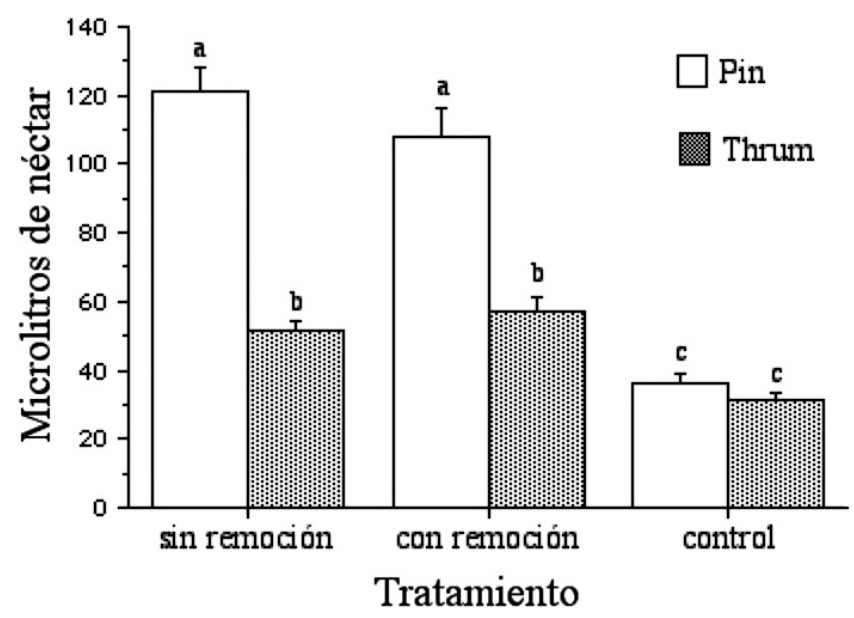

Figura 1. Variación (media \pm e.e) en los volúmenes de néctar (microlitros) colectados en flores de ambos morfos y sometidos a cada tratamiento. Letras distintas denotan diferencias significativas $P<0.05$. 
ambos morfos presentan porcentajes de azúcar similares $\left(F_{1,84}=0.205, P=0.651\right)$ y ambos morfos mantienen este patrón al aplicarles los tratamientos experimentales $\left(F_{2,84}=\right.$ $0.184, P=0.8325)$. Sin embargo, independientemente del morfo, las plantas sometidas a remoción constante de néctar presentaron los mayores valores de porcentaje de azúcar $\left(F_{2,84}=229.817, P=0.0001\right.$, Figura 2$)$.

Los mayores valores de concentración, independientemente del morfo, fueron registrados al final de la temporada $\left(F_{1,84}=23287.170, P=0.0001\right)$, y particularmente para el tratamiento de remoción $\left(F_{2,84}=100.641, P=0.0001\right)$. Tanto la interacción temporada $\times$ morfo $(F=0.002, P=$ $0.965)$ como la interacción temporada $\times$ morfo $\times$ tratamiento $\left(F_{2,84}=0.330, P=0.967\right)$ no fueron estadísticamente significativas.

Compensación de flores. El análisis del número total de flores producido a través de 14 conteos, a partir de la última semana del mes de mayo hasta la segunda semana del mes de agosto del 2009, sugiere que no hay diferencias significativas entre morfos $\left(F_{1,168}=1.428, P=0.2552\right)$, tratamientos $\left(F_{2,168}=0.072, P=0.930\right)$, y su interacción $\left(F_{2,168}=0.104, P\right.$ $=0.9019)$. De igual forma, no hubo diferencias significativas entre las interacciones conteos $\times$ morfo $\left(F_{14,168}=1.422\right.$, $P=0.1422)$, conteos $\times$ tratamiento $\left(F_{28,168}=0.718, P=\right.$ $0.883)$ y conteo $\times$ morfo $\times$ tratamiento $\left(F_{28,168}=0.361, P=\right.$ 0.988). En cambio, sí hubo diferencias significativas entre el número de flores cuantificado en cada uno de los conteos $\left(F_{14,168}=49.577, P=0.0001\right)$.

Cuantificación del número de semillas producidas. Las semillas colectadas en todos los tratamientos fueron viables. El número de semillas cuantificado, independientemente del tratamiento aplicado, demostró que las plantas del morfo thrum producen más semillas que las del morfo pin $\left(F_{1,156}=\right.$ $4.543, P=0.034)$. Asimismo, independientemente del mor-

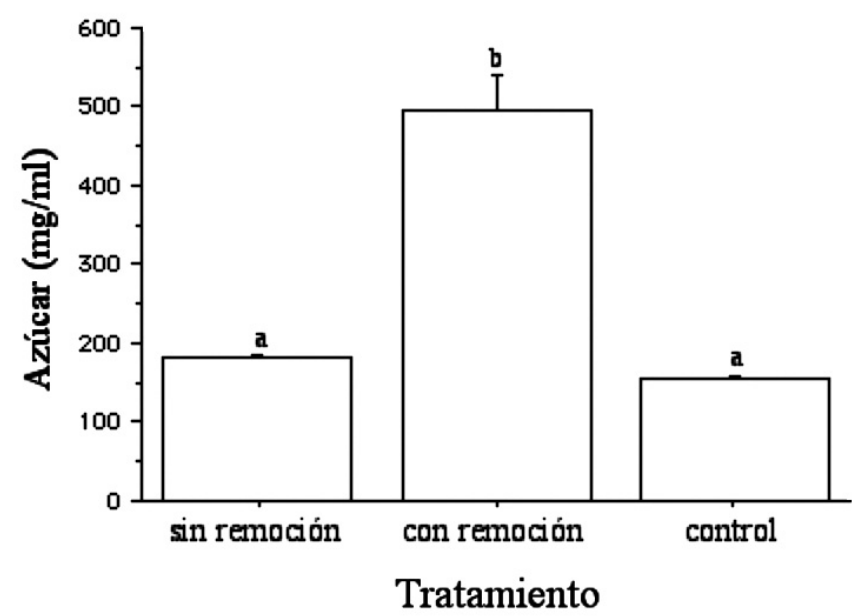

Figura 2. Concentración de azúcar (media \pm e.e) del néctar colectado en plantas (independientemente del morfo floral) sometidas a los distintos tratamientos. Letras distintas denotan diferencias significativas $P<0.05$. fo, las plantas con y sin remoción produjeron más semillas que las plantas del tratamiento control $\left(F_{2,156}=32.501, P=\right.$ $0.0001)$; sin embargo, fueron las plantas del morfo thrum las que produjeron más semillas que las pin, particularmente en el tratamiento sin remoción y el control $\left(F_{2,156}=12.025\right.$, $P=0.0001$, Figura 3).

\section{Discusión}

En este trabajo se encontró que Bouvardia tiene la capacidad de producir grandes cantidades de néctar (y azúcar) cuando es sometida a niveles altos de remoción (simulando robo de néctar), sin tener aparentemente un costo en cuanto a la producción de semillas. Este patrón apoya nuestra hipótesis de que las plantas sometidas a robo de néctar pueden compensar el néctar extraído, lo cual puede ser análogo a un mecanismo de tolerancia. Por el contrario, la simulación de robo no tuvo ningún efecto sobre la producción de flores.

El robo de néctar es un fenómeno común en las angiospermas (Irwin y Maloof, 2002). Usualmente los organismos que obtienen esta recompensa de forma ilegítima, pueden afectar directa (p. ej. dañando los órganos reproductivos florales) o indirectamente (modificando la conducta de los polinizadores) a las plantas (Irwin y Brody, 2000; Adler et al., 2001, Lara y Ornelas, 2002a). De forma análoga a lo que ocurre con la herbivoría, las plantas sometidas a robo de néctar están continuamente sujetas a un ataque (en este caso la extracción de esta recompensa), y pueden desarrollar resistencia (habilidad de las plantas para disminuir la frecuencia del ataque) a ellos. Diversos mecanismos han sido propuestos como formas de resistencia al robo de néctar, los cuales incluyen la presencia de toxinas o deterrentes en el néctar (Adler, 2000), la producción de néctar en horarios distintos a la actividad de los ladrones (Irwin et al., 2001), el

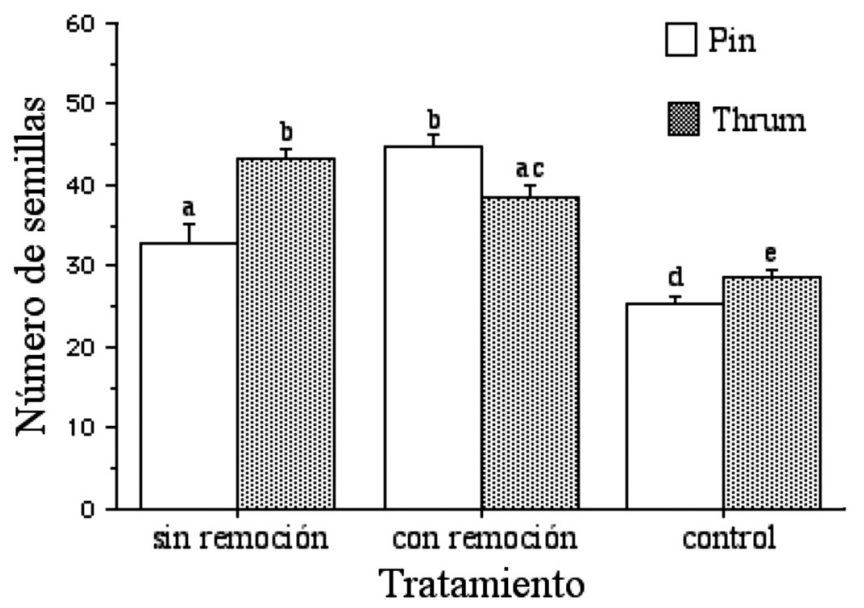

Figura 3. Número de semillas (media \pm e.e) cuantificado para frutos en plantas de ambos morfos y sometidas a los diferentes tratamientos. Letras distintas denotan diferencias significativas $P<0.05$. 
crecimiento junto a especies de plantas con mayor incidencia de robo de néctar (Irwin y Brody, 2000) y la presencia de brácteas o tubos florales cortos que contribuyen a evitar a los ladrones (Proctor et al., 1996; Lara y Ornelas, 2001a, b). Sin embargo, la resistencia representa sólo una estrategia que las plantas pueden utilizar para contrarrestar los ataques de los ladrones de néctar, pudiendo éstas incluso tolerarlos.

El efecto negativo de los ladrones puede ser tolerable, si por ejemplo, tal como fue propuesto por Irwin et al. (2008), las plantas pueden producir suficiente néctar para alimentar a los visitantes (polinizadores y ladrones). Éste es el caso de Bouvardia, pues plantas de ambos morfos sometidas a niveles constantes de remoción de néctar tuvieron volúmenes similares a las plantas sin la simulación de robo. Este patrón de sobreproducir néctar en plantas sometidas artificialmente a robo ha sido únicamente probado en Ipomopsis aggregata (Polemoniaceae; Irwin et al., 2008; Irwin, 2009) y sus resultados obtenidos conllevan a interesantes similitudes y se complementan con los datos obtenidos en nuestro trabajo. Por ejemplo, tanto en Bouvardia como en Ipomopsis, las plantas parecen sobrecompensar el néctar extraído artificialmente, llevando a la obtención de volúmenes similares con respecto a las plantas sin extracción simulada por robo. Ésta indica que las plantas de ambas especies pueden tolerar el robo de néctar, compensando la recompensa perdida por robo e igualando los niveles de oferta para los polinizadores.

Nuestros resultados demostraron que la tolerancia al robo de néctar es diferencial entre los morfos florales de Bouvardia. El morfo pin (longiestilado) compensó mayores volúmenes de néctar (incluso más concentrados) que el morfo thrum (breviestilado). Casos de especialización sexual basada en la producción y oferta de néctar han sido poco explorados en especies heteroestílicas. Resultados contrastantes en especies diestílicas muestran que los patrones de producción de néctar pueden no diferir entre los morfos florales en la cantidad y calidad de la recompensa ofrecida a los polinizadores (Palicourea petiolaris y P.fendleri, Sobrevila et al., 1983; Fernandusa speciosa, Castro y Oliveira, 2001; Psychotria poepigiana, Coelho y Barbosa, 2004; P. barbiflora, Teixeira y Machado 2004a, b; Palicourea demissa, Valois-Cuesta et al., 2011), aunque otros estudios han mostrado diferencias (Palicourea padifolia, Ornelas et al., 2004, Fagopirum esculentum, Cawoy et al., 2008). Una oferta de néctar diferencial entre los morfos florales quizás podría generar tasas diferenciales de visita y de deposición de polen (Valois-Cuesta et al., 2011), y eventualmente, ello podría acarrear consecuencias negativas sobre el éxito reproductivo de las plantas (Manetas y Petropoulou, 2000). En nuestro estudio, las plantas de ambos morfos y sometidas a tratamientos de remoción fueron polinizadas artificialmente, sin evaluar los patrones de visita por polinizadores; por ello nuestros resultados sólo pueden ser conclusivos con respecto a la compensación diferencial de néctar entre morfos.
El resultado encontrado con respecto al hecho de que las flores polinizadas manualmente en plantas control, produjeron menos semillas que aquellas flores en plantas sometidas a tratamiento de remoción y no remoción de néctar es intrigante. Dado que a lo largo del estudio la planta control se mantuvo expuesta a todo tipo de visitante y sus flores no sometidas a remoción artificial, es posible que el costo acumulado por estos niveles de remoción natural fueran mayores a los costos en aquellas plantas que se mantuvieron cubiertas a lo largo del estudio (y sin remoción), y las plantas expuestas pero sometidas a remoción artificial. Sin embargo, esta posibilidad requiere una futura comprobación.

Con respecto al papel de la producción de flores en respuesta al robo de néctar, los estudios realizados en Ipomopsis indican que las plantas con altos niveles de robo presentan una mayor producción de flores, sugiriendo una mayor habilidad para compensar los costos del robo vía la función masculina de donación de polen (Irwin et al., 2008; Irwin, 2009). Sin embargo, en nuestro estudio no se encontró compensación de flores, pero a diferencia de los mencionados estudios, la compensación parece mostrarse a nivel de producción de semillas, sugiriendo una tolerancia vía la función femenina en estas plantas. De esta manera, las flores pin (longiestiladas) son las que tuvieron mayor producción de semillas en condiciones de simulación de néctar. Diferencias previas entre morfos en especies diestílicas en atributos a nivel de frutos y semillas han sido reportadas para Palicourea padifolia, donde las plantas longiestiladas producen frutos más grandes que las plantas breviestiladas (Contreras y Ornelas, 1999; Ornelas et al., 2004).

Las razones del por qué los morfos difieren en el tamaño o número de los frutos y/o semillas, no son claras. Sin embargo, algunos autores han encontrado que la masa de los frutos decrece conforme aumenta la proporción de daño foliar (Ornelas et al., 2004). A partir de esta evidencia, inferimos que tasas diferenciales de herbivoría entre los morfos, quizás podrían generar diferencias en la eficiencia fotosintética de cada uno de ellos y, en consecuencia, los morfos presentarían desigualdad en su capacidad de asignar asimilados fotosintéticos destinados al desarrollo de sus frutos y semillas. En el caso del robo, es posible que las plantas sometidas a esta repetida remoción sean capaces de incrementar la disponibilidad de recursos hacia las flores con robo, permitiendo compensar la pérdida de volumen. Energéticamente hablando, esta posibilidad es viable si consideramos que a diferencia de las flores, el néctar no tiene un valor fotosintético y su producción puede ser incluso no costosa en los términos mencionados (Ordano y Ornelas, 2005).

La evidencia recabada tanto en Ipomopsis como en Bouvardia, apoya la idea de que las plantas sometidas a robo de néctar pueden ser capaces de asignar diferencialmente recursos para compensar los costos de esta extracción. La aplicación de tratamientos experimentales a nivel de planta (usando todas las flores producidas a lo largo del periodo 
de floración), ofrece la posibilidad de controlar posibles efectos fenológicos, y con ello simular los efectos aditivos y acumulados que soportan las plantas en condiciones naturales. Aún cuando desconocemos el papel de la variación genotípica en los resutados obtenidos, nuestros resultados ofrecen una aproximación al poco explorado fenómeno de la tolerancia hacia el robo de néctar, y resaltan la necesidad de un mayor número de estudios en otros sistemas naturales para determinar la generalidad hasta ahora obtenida.

\section{Agradecimientos}

Agradecemos los comentarios de Jafet M. Nassar y un revisor anónimo. A la Caseta 5 de la Coordinación General de Ecología del Estado de Tlaxcala por las facilidades otorgadas. Asimismo, apreciamos el apoyo en el trabajo de campo de Israel Morales y Cristina Tello.

\section{Literatura citada}

Adler L.S. 2000. The ecological significance of toxic nectar. Oikos 91:409-420.

Adler L.S., Karban R. y Strauss S.Y. 2001. Direct and indirect effects of alkaloids on plant fitness via herbivory and pollination. Ecology 82:2032-2044.

Brody A.K. y Mitchell R.J. 1997. Effects of experimental manipulation of inflorescence size on pollination and pre-dispersal seed predation in the hummingbird-pollinated plant Ipomopsis aggregata. Oecologia 110:86-93.

Castro C.C. y Oliveira P.E.A. 2001. Reproductive biology of the protandrous Ferdinandusa speciosa Pohl (Rubiaceae) in southeastern Brazil. Revista Brasileña de Botánica 24:167172.

Cawoy V., Kinet J.M. y Jacquemart A.L. 2008. Morphology of nectaries and biology of nectar production in the distylous species Fagopyrum esculentum. Annals of Botany 102: 675-684.

Coelho C.P. y Barbosa A.A. 2004. Biologia reproductiva de Psychotria poeppigiana Mull. Arg. (Rubiaceae) em mata de galeria. Acta Botánica Brasileña 18:481-489.

Contreras P.S. y Ornelas J.F. 1999. Reproductive conflicts of Palicourea padifolia (Rubiaceae), a distylous shrub of a tropical cloud forest in Mexico. Plant Systematic and Evolution 219:225-241.

Díaz-Ojeda E.V. 1992. Informe del Parque Nacional La Malinche. Jefatura del Programa Forestal de Tlaxcala. Gobierno del Estado, Tlaxcala.

Faivre A.E. 2000. Ontogenetic differences in heterostylous plant and implications for development from a herkogamous ancestor. Evolution 54:847-858.

Hernandez-Guerrero, A. 2006. Colibríes y la especialización sexual en el arbusto distílico Bouvardia ternifolia (Rubiaceae). Tesis de licenciatura. UNAM, Facultad de Ciencias. México, D.F. 45 pp.

Howe G.A., Lightner J., Browse J. y Ryan C.A. 1996. An octadecanoid pathway mutant (JL5) of tomato is compromised in signaling for defense against insect attack. Plant Cell 8:20672077.

Irwin R.E. 2009. Realized tolerance to nectar robbing: compensa- tion to floral enemies in Ipomopsis aggregata. Annals of Botany 103:1425-1433.

Irwin R.E. y Brody A.K. 2000. Consequences of nectar robbing for realized male function in a hummingbird-pollinated plant. Ecology 81:2637-2643.

Irwin R.E., Brody A.K. y Waser N.M. 2001. The impact of floral larceny on individuals, population, and communities. Oecologia 129:161-168.

Irwin, R.E. y Maloof J.E. 2002. Variation in nectar robbing over time, space, and species. Oecologia 133:525-533.

Irwin R.E., Lynn S.A. y Brody A.K. 2004. The dual role of floral traits: Pollinator attraction and plant defense. Ecology 85:15031511.

Irwin R.E., Galen C., Rabenold J.J., Kaczorowski R. y McCutcheon M.L. 2008. Mechanisms of tolerance to floral larceny in two wildflower species. Ecology 89:3093-3104.

Kearns C.A. y Inouye D.W. 1993. Techniques for Pollination Biologists. University Press of Colorado, Niwot.

Lara C. 2006. Temporal dynamics of flower use by hummingbirds in a highland temperate forest in Mexico. Ecoscience 13:23-29.

Lara C. y Ornelas J. 2001a. Preferential nectar robbing of flowers with long corollas: experimental studies of two hummingbirds visiting three plant species. Oecologia 128:263-273.

Lara C. y Ornelas J.F. 2001b. Nectar "theft" by hummingbird flowers mites and its consequences for seed set in Moussonia deppeana. Functional Ecology 15:78-84.

Lara C. y Ornelas J.F. 2002a. Effects of nectar theft by flower mites on hummingbird behavior and the reproductive success of their host plant, Moussonia deppeana (Gesneriaceae). Oikos 96:470-480.

Lara C. y Ornelas J.F. 2002b. Flower mites and nectar production in six hummingbird-pollinated plants with contrasting flower longevities. Canadian Journal of Botany 80:1216-1229.

Lara C. y Ornelas J.F. 2003. Hummingbirds as vectors of fungal spores in Moussonia deppeana (Gesneriaceae): taking advantage of a mutualism? American Journal of Botany 90:262-269.

Maloof J.E. y Inouye D.W. 2000. Are nectar robbers cheaters or mutualists? Ecology 81:2651-2661.

Manetas Y. y Petropoulou Y. 2000. Nectar amount, pollinator visit duration and pollination success in the mediterranean shrub Cistus creticus. Annals of Botany 86:815-820.

Ordano M. y Ornelas J.F. 2005. The cost of nectar replenishment in two epiphytic bromeliads. Journal of Tropical Ecology 21:541547

Ornelas J.F., González C., Jiménez L., Lara C. y Martínez A.J. 2004. Reproductive ecology of distylous Palicourea padifolia (Rubiaceae) in a tropical montane cloud forest. II. Attracting and rewarding mutualistic and antagonistic visitors. American Journal of Botany 91:1061-1069.

Paige K.N. y Whitham T.G. 1987. Overcompensation in response to mammalian herbivory: the advantage of being eaten. The American Naturalist 129:407-416.

Proctor M., Yeo P. y Lack A. 1996. The Natural History of Pollination. Timber Press, Portland.

Roy B.A. y Kirchner J.W. 2000. Evolutionary dynamics of pathogen resistance and tolerance. Evolution 54:51-63.

Salinas-Esquivel, L. 2007. Robo de néctar y sus efectos en la interacción entre colibríes y Bouvardia ternifolia (Rubiaceae): pruebas experimentales. Tesis de licenciatura. UNAM, Facultad de Ciencias. México, D. F. pp 27. 
Sobrevila C., Ramirez N. y de Enrech N.X. 1983. Reproductive biology of Palicourea flendleri and Palicourea petiolaris (Rubiaaceae), heterostylous shrubs of a tropical cloud forest in Venezuela. Biotropica 15:161-169.

Strauss S.Y. y Agrawal A.A. 1999. The ecology and evolution of plant tolerance to herbivory. Trends in Ecology and Evolution 14:179-185.

Strauss S.Y., Rudgers J.A., Lau J.A. y Irwin R.E. 2002. Direct and ecological costs of resistance to herbivory. Trends in Ecology and Evolution 17:278-285.

Strauss S.Y. y Irwin R.E. 2004. Ecological and evolutionary consequences of multispecies plant-animal interaction. Annual Review of Ecology, Evolution and Systematics 35:435-466.

Teixeira L.A.G. y Machado I.C. 2004a. Biologia da polinização e sistema reproductivo de Psychotria barbiflora DC. (Rubiaceae). Acta Botánica Brasileña 18:853-862.

Teixeira, L.A.G. y Machado I.C. 2004b. Sabicea cinerea Aubl.
(Rubiaceae): distilia e polinização em um fragmento de floresta Atlântica em Pernambuco, Nordeste do Brasil. Revista Brasileña de Botánica 27:193-204.

Torres-Díaz I. 2007. Efecto de la herbivoría en la interacción entre colibríes y el arbusto distílico Bouvardia ternifolia (Rubiaceae): Experimentos de campo en La Malinche, Tlaxcala. Tesis de Licenciatura. UNAM, Facultad de Ciencias. México, D. F. pp. 45.

Torres I., Salinas L., Castillo-Guevara C. y Lara C. 2008. Antagonist and their effects in a hummingbird-plant interaction: Field experiments. Ecoscience 15:65-72.

Valois-Cuesta H., Soriano P. J. y Ornelas J.F. 2011. Asymmetrical legitimate pollination in distylous Palicourea demissa (Rubiaceae): the role of nectar production and pollinator visitation. Journal of Tropical Ecology 27:393-404.

Zar J.H. 1999. Biostatistical Analysis. Cuarta edición. Prentice Hall Inc., Nueva Jersey.

Recibido: 17 de marzo de 2012

Aceptado: 26 de julio de 2012 